\title{
Diverse 3D Microarchitectures Made by Capillary Forming of Carbon Nanotubes
}

\author{
By Michael De Volder, Sameh H. Tawfick, Sei Jin Park, Davor Copic, Zhouzhou Zhao, \\ Wei Lu, and A. John Hart*
}

Scalable fabrication of complex and multifunctional surface architectures is essential to future advances in metamaterials, ${ }^{[1]}$ substrates for cell culture and tissue engineering, ${ }^{[2]}$ dynamic surfaces, ${ }^{[3]}$ and lab-on-a-chip systems. ${ }^{[4]}$ Nevertheless, it remains difficult to create non-planar, curved, re-entrant, and other freeform geometries at sub-millimeter scales; and existing methods of fabricating three-dimensional (3D) microstructures suffer from tradeoffs among feature geometry, heterogeneity, resolution, and throughput. Serial methods such as stereolithography, ${ }^{[5]}$ multiphoton lithography, ${ }^{[6]}$ and focused ion beam processing $^{[7]}$ can create arbitrary forms; however, these are only practical for fabricating master templates or small batches. Parallel methods are typically limited to creating arrays of identical structures, such as by interference or inclined exposure lithography. ${ }^{[8]}$ An additional limitation is that current techniques typically utilize polymers that have low mechanical stiffness and strength, and low thermal and electrical conductivities. Further, these methods largely do not enable integration of organized nanostructures.

Synthetic self-assembly ${ }^{[9]}$ is an emerging means to complement top-down fabrication by control of interactions among nanostructures, and between nanostructures and physical templates. Nature uses self-assembly to create complex materials such as tissues and skeletal structures ${ }^{[10]}$ having inherent anisotropy that creates directional properties that are largely not yet achieved in synthetic materials. Despite rapid advances in the synthesis of nanostructures, and in their assembly using specific (e.g., DNA) and non-specific (e.g., electrostatic and van der Waals) interactions, ${ }^{[11]}$ a persistent limitation is that synthetic self-assembly typically lacks deterministic control of shape at a length scale far exceeding that of the constituent building block.

We present a methodology for the construction of robust threedimensional (3D) assemblies of filamentary nanostructures,

[*] Dr. M. De Volder, S. H. Tawfick, S. J. Park, D. Copic, Z. Zhao, Prof. W. Lu, Prof. A. J. Hart

Department of Mechanical Engineering

University of Michigan

2350 Hayward Street

Ann Arbor, MI 48109 (USA)

E-mail: ajohnh@umich.edu

Dr. M. D. Volder

IMEC, Kapeldreef 75, 3001 Heverlee, Belgium

Department of Mechanical Engineering

Katholieke Universiteit Leuven

3001 Leuven (Belgium)

DOI: 10.1002/adma.201001893 whereby lithographically defined patterns of vertically aligned filaments are transformed into 3D shapes by self-directed capillary action which is initiated by liquid condensation. We demonstrate this method, which we call "capillary forming", via fabrication of a diverse library of carbon nanotube (CNT) microarchitectures. Further, we demonstrate that capillary formed CNT structures have exceptional robustness and can be used as molds for mass-production of 3D polymer structures. This new approach to manipulate nanoscale filaments using local mechanical deformations facilitates deterministic design and fabrication of 3D microarchitectures having complex geometries along with nanotextured surfaces, yet requires only standard two-dimensional patterning and ambient pressure thermal processing.

Our approach to capillary forming of CNTs is depicted in Figure 1A. First, a film of iron catalyst is patterned by optical lithography on a silicon wafer substrate. Second, microstructures made of vertically aligned CNTs (CNT "forests") are grown by atmospheric pressure thermal chemical vapor deposition (CVD). ${ }^{[12,13]}$ Next, a solvent such as acetone is condensed on the substrate. This is done by inverting the substrate with CNTs over a beaker containing a boiling solvent such as acetone (Figure S1 of the Supporting Information). The solvent vapor rises through the beaker and condenses on the substrate which remains cool. Due to capillary rise, the solvent is drawn into each CNT microstructure independently. After the substrate has been exposed to the vapor stream for the desired duration, the substrate is removed from the beaker and the liquid is evaporated under ambient conditions. During infiltration and evaporation of the liquid, the CNTs within each structure densify, and each structure is shaped individually by the forces resulting from capillary action.

In past studies, surface tension has been utilized to aggregate wet hair, ${ }^{[14]}$ microfibers, ${ }^{[15]}$ and polymer pillars. ${ }^{[16]}$ Specifically, immersion of CNT forests in liquids has been used to segregate CNT forests into cellular foams, ${ }^{[17,18]}$ and to achieve isotropic contraction ${ }^{[19]}$ and unidirectional toppling ${ }^{[20]}$ of CNT microstructures. Although elastocapillary aggregation ${ }^{[14]}$ occurs locally within filamentary networks regardless of how the liquid is delivered, we found critical difference between the well-known immersion method and our method of liquid delivery by condensation. As a substrate with CNT microstructures is immersed in liquid, the meniscus creates lateral forces that draw structures toward the substrate. ${ }^{[20]}$ Further, the movement of menisci between structures during evaporation can cause the structures to undesirably aggregate, which limits the size and spacing of individual microstructures that can remain vertical after the process. On the other hand, condensation of 

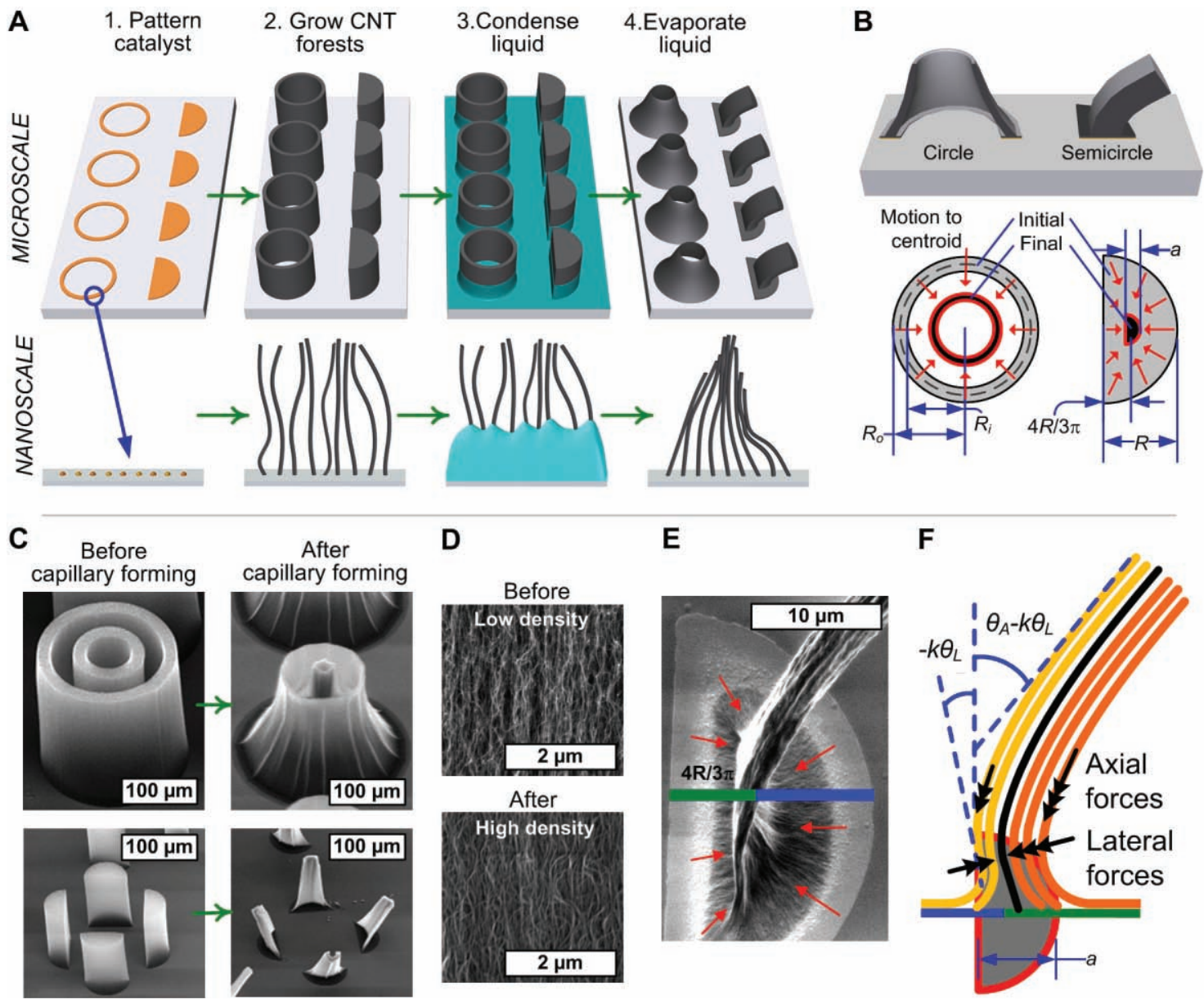

Figure 1. Method and mechanism of capillary forming of CNT forest microstructures. (A) Illustration of CNT forest growth and capillary forming sequence. (B) Schematics of CNT microarchitectures made by capillary forming of initially circular and semicircular CNT forests. (C) SEM images of hollow cylindrical and semicylindrical CNT forests before and after capillary forming. (D) Change in CNT packing density due to capillary forming. (E) Alignment of CNTs pointing toward the centroid of an initially semicylindrical CNT forest. (F) Schematic of lateral and axial forces during capillary forming of a semicylindrical forest.

the liquid isolates the surface tension forces within each CNT microstructure because the liquid infiltrates each structure individually.

As a result of these differences, we found that immersion detrimentally couples and flattens small-footprint CNT (e.g., $<400 \mu \mathrm{m}^{2}$ ) and high aspect ratio (e.g., height/width $>3$ ) structures, yet condensation allows the structures to be densified individually. This comparison is shown in Figure S2. Ex situ examination of structures on which capillary forming is terminated prematurely (Figure S3) verifies that densification occurs from the base of the CNT forests. Imaging the capillary forming process in situ at $\approx 10$ Torr using environmental scanning electron microscopy (ESEM), shown in Video S1, reveals that the CNTs densify both during infiltration and evaporation of the liquid under low-pressure conditions. After capillary forming, the final shape of the densified CNT structure is held by intermolecular attractive forces, as well as mechanical interlocking due to the waviness of the aligned CNTs (Figure 1D).

Decoupling of the capillary forces by self-directed infiltration facilitates design of the CNT growth pattern to achieve a deterministic transformation from prismatic forests to intricate and robust 3D shapes. This transformation is governed by the distribution of lateral forces (locally perpendicular to the CNTs) and axial forces (locally parallel to the CNTs) that occurs within each structure during capillary forming. Surface tension causes the CNTs to aggregate locally according to the elastocapillary mechanism, and the forest globally contracts toward the centroid of its cross-sectional shape. Thus, for a circle, the contraction is toward the center; while for a semicircle, the contraction is toward the point at a distance $4 R / 3 \pi$ from the straight edge of the semicircle (Figure 1B), which is seen in an SEM image of the base (Figure 1E) of the structure. As this contraction occurs, the CNTs near the substrate are pulled inward toward the centroid, and this in turn pulls down on the upper portions of the forest. For circles, the force distribution is axisymmetric and the final structure therefore slopes toward its apex. For semicircles, the force distribution is asymmetric due to the asymmetric location of the centroid. This causes the structure to deflect laterally, creating a curved beam. 
The distribution of forces (Figure 1F) is further understood by examining capillary forming of semicylindrical CNT forests. The capillary pressure during densification introduces lateral forces on the structure, which are proportional to the number of CNTs on either side of the centroid (Figure S4). A net bending moment results and causes the structure to deflect $\left(\theta_{\mathrm{L}}\right)$ toward the straight edge of the semicircle. Additionally, because of mechanical coupling between the base and the upper portion of the forest, the lateral motion of the CNTs creates a distribution of axial pulling forces (Figure S5), which acts on the CNTs above the substrate. The axial forces thus create another bending moment at the base, which causes the structure to deflect $\left(\theta_{\mathrm{A}}\right)$ toward the curved edge of the cross-section. As discussed in the supporting information, the net deflection angle is calculated by modeling the structure as a cantilever beam, and adding the contributions due to the lateral and axial forces, $\theta=-\theta_{\mathrm{L}}+k \theta_{\mathrm{A}}$. The relaxation factor $k(0<k<1)$ accounts for slip among the CNTs during capillary forming, which partially relaxes the bending moment and reduces the lateral deflection.

As shown in Figure 2A and Figure 2B, the deflection angle of curved beams made by capillary forming of semiannular CNT forests depends precisely on the catalyst pattern design parameter $r$, where $r=R_{\mathrm{i}} / R_{\mathrm{o}}$ Comparison between the analytical model and measurements also verifies that the deflection angle, and therefore the balance between lateral and axial forces, depends on the amount of slip among the CNTs during capillary forming. Slip can be controlled by plasma etching the top surface of the CNT forests before capillary forming.

In comparison, design of annular catalyst patterns facilitates fabrication of sloped CNT micro-wells, which experience azimuthally symmetric force distributions during capillary forming. The displacement of the CNTs toward the center of the annulus depends on the wall thickness of the annulus, as shown in Figure 2C and Figure 2D. Thin-walled annuli fold inward and onto the substrate, creating circular thin films of radially oriented CNTs, while thick-walled annuli remain straight. The array of sloped micro-wells emphasizes the ability to simultaneously fabricate structures having different shapes, and CNT organizations ranging from parallel to vertical to the substrate.

Additional catalyst shapes and arrangements of shapes have been transformed into complex micro-scale topologies which are difficult or impossible to make using known fabrication approaches. For instance, as shown in Figure 3A, concentric annuli can be designed to form overhanging wells having different slopes, or radially-aligned sheets surrounding vertical needles. Circular arrangements of bending structures resemble arrangements of flower petals, which can be designed to bend inward or outward from a common point (Figure 3B). Intricate micro-helices with deterministic handedness are formed from shapes comprising semicircles merged with a thin annulus (Figure 3C). These catalyst shapes combine the elementary motions of contraction and bending, and the helical angle and

A
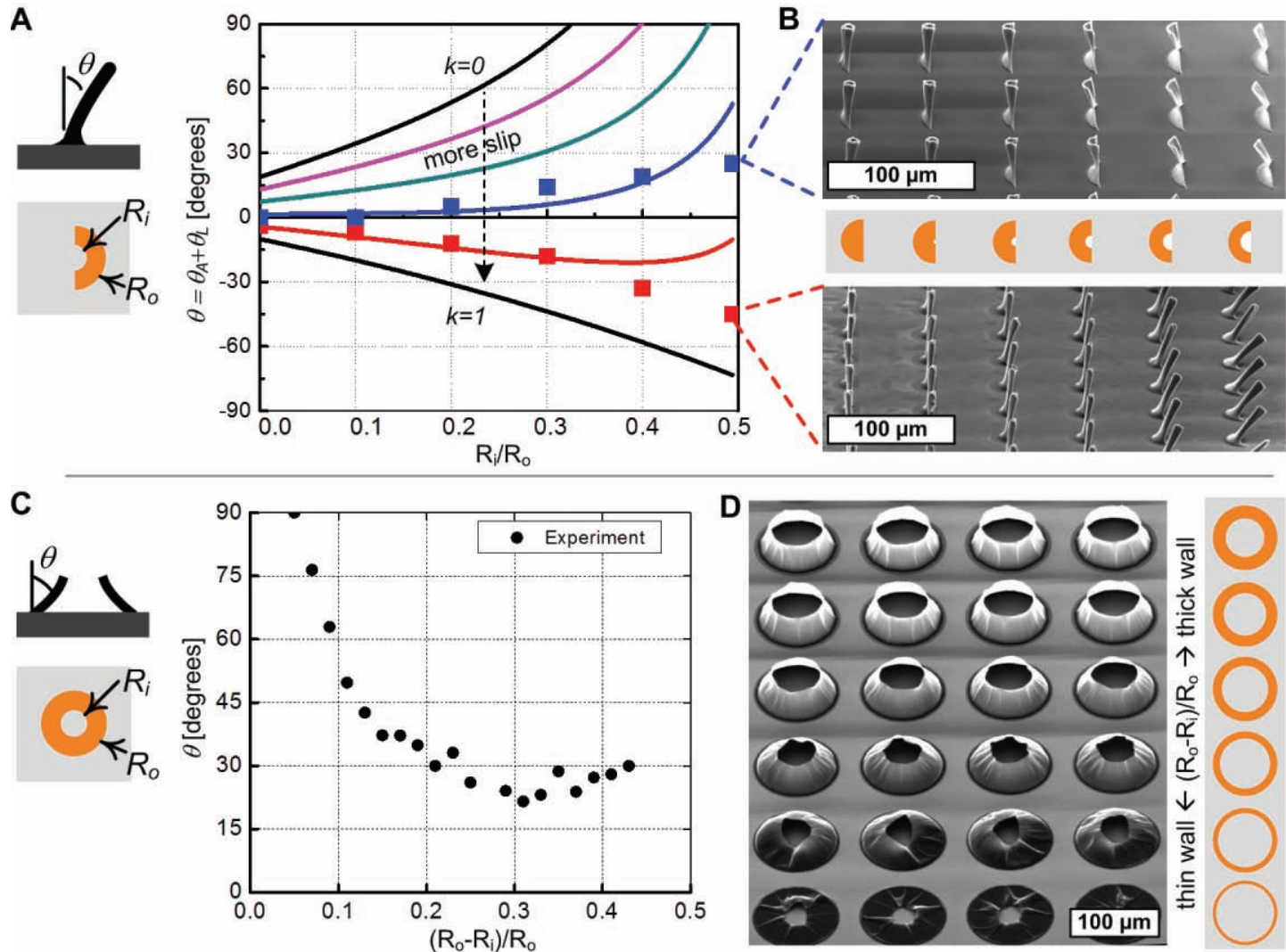

Figure 2. Modeling of capillary forming and spatial control by catalyst shape design. (A) Analytical prediction of deflection angle of capillary formed semicylinders, and comparison to measured angles of structures shown in (B). (C) Measured and predicted sidewall slope angles of capillary formed cylindrical forests, corresponding to the array of heterogeneous structures shown in (D). 
A
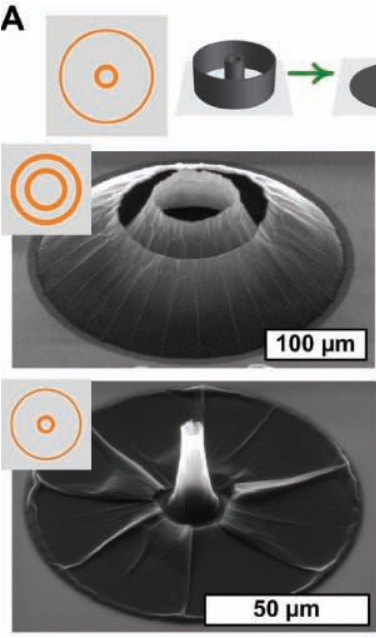

B
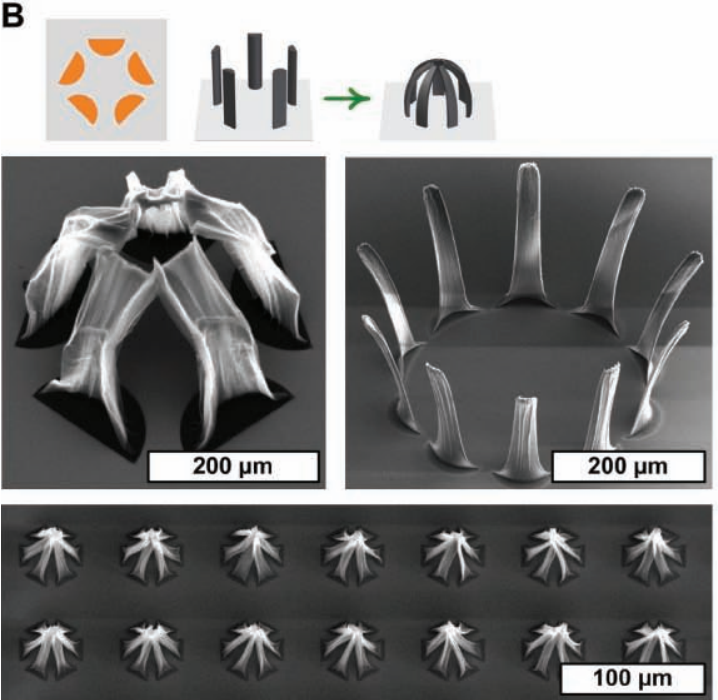

C
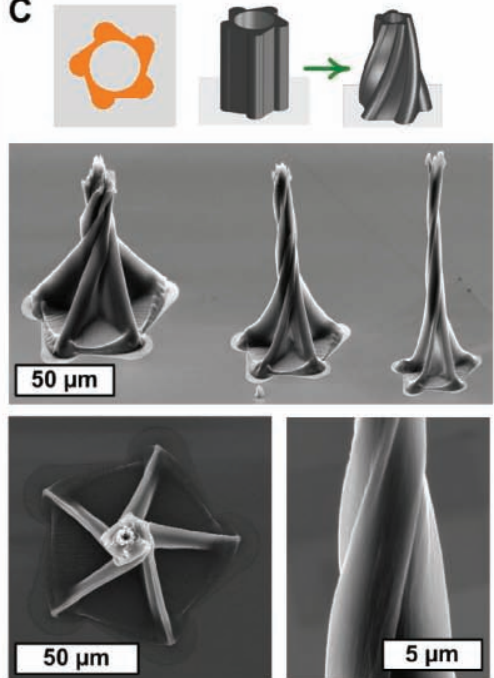

$50 \mu \mathrm{m}$
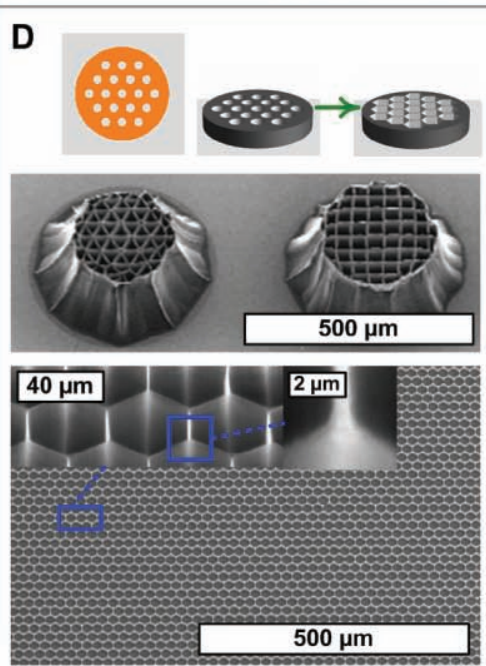
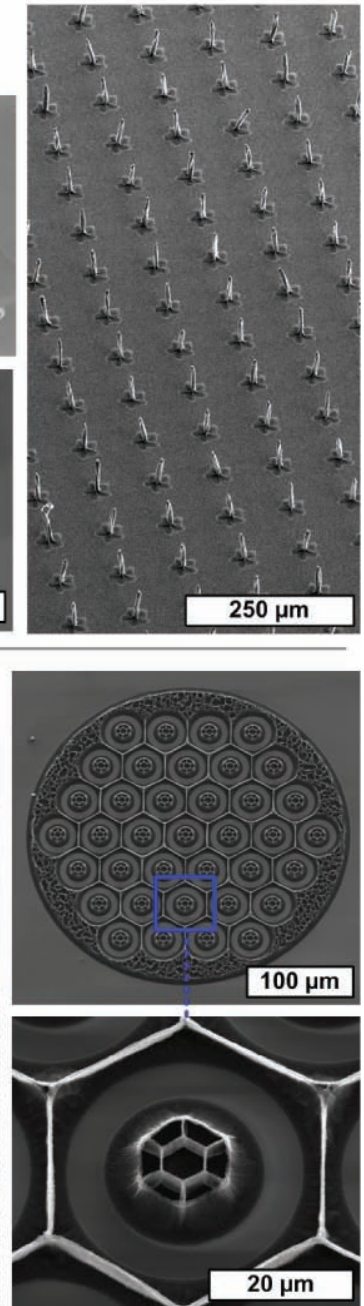

Figure 3. Complex CNT microarchitectures and multi-directional patterns created by coupling elementary catalyst shape building blocks. (A) Concentric microwells with different slopes, and an array of structures each comprising a radially oriented CNT film surrounding a vertical needle. (B) "Blooming flowers" of radially-oriented bending CNT structures. (C) CNT micro-helices with deterministic handedness and pitch. (D) Thin-walled lattices, formed from CNT forests with patterned internal voids.

pitch of the final structure are determined by the dimensions of the catalyst shape. As a last example, solid CNT forests with arrays of small holes are transformed to well-ordered cellular structures ${ }^{[17]}$ with sub-micron wall thickness (Figure 3D).

The final shape of a CNT microarchitecture made by capillary forming depends on the geometry, dimensions, and structural characteristics of the starting CNT forest. By tuning the CNT diameter and areal density by changing the duration of catalyst annealing prior to CNT growth, and by etching the tangled "crust" from the top of the CNT forest prior to capillary forming, we have controlled the densification factor ${ }^{[25]}$ from approximately 5 to 30 . The latter represents a bulk density of approximately $0.50 \mathrm{~g} / \mathrm{cm}^{3}$, compared to $1.26 \mathrm{~g} / \mathrm{cm}^{3}$ for ideally packed CNTs with these dimensions. Even with a simple apparatus for condensing the solvent, we have achieved highly uniform results on substrates as large as $20 \mathrm{~mm} \times 20 \mathrm{~mm}$, and this size is currently limited by the size of our CNT growth furnace.
Importantly, the low as-grown density (typically 1-5\%) of CNT forests gives bulk properties far beneath those of existing microfabrication materials ${ }^{[21]}$ and renders as-grown CNT forests incompatible with lithographic processing. However, the measured Young's modulus of a capillary formed CNT (CF-CNT) cylinder (Figure $4 \mathrm{~B}$ ) is $E=5 \mathrm{GPa}$, which is a $\approx 100$-fold enhancement over the as-grown CNT forest $(E=54 \mathrm{MPa})$. These values were measured by compression testing using a flat diamond punch mounted in a nanoindentation machine (Figure 4A), giving the loading curves shown in Figure 4C.

Further, CF-CNT structures can be infiltrated with polymers by spin-coating, enabling self-directed fabrication of nanocomposite microstructures. Structures made by infiltration of SU-8 and PMMA into CF-CNT cylinders have $E=18 \mathrm{GPa}$ and $E=25 \mathrm{GPa}$, respectively. These values are 5- to 10 -fold enhancements over pure SU-8 $(E=2-4 \mathrm{GPa})$ and pure PMMA $(E=2-5 \mathrm{GPa})$. To our knowledge, these are the highest values 
A

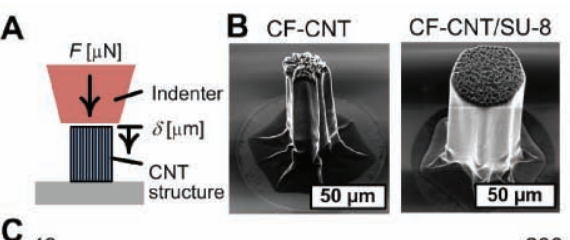

C

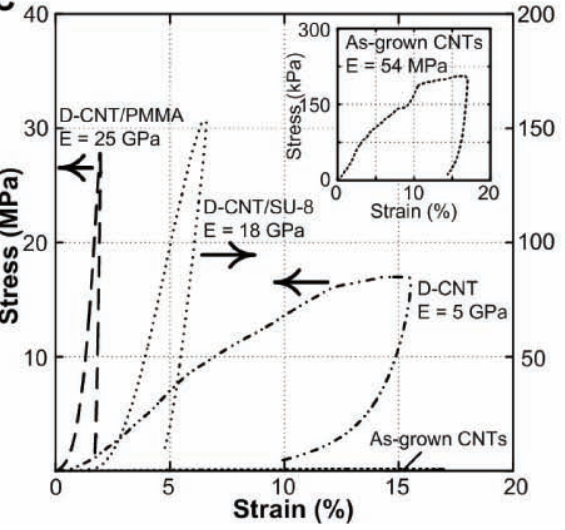

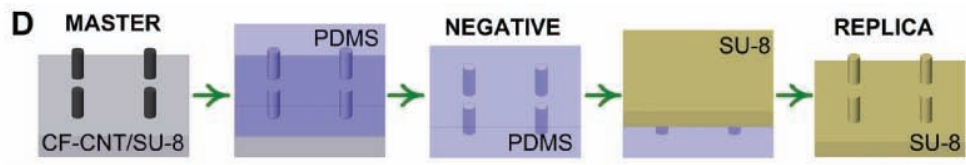

E

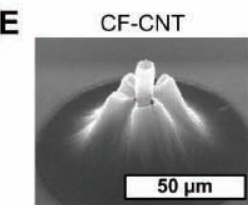

F master
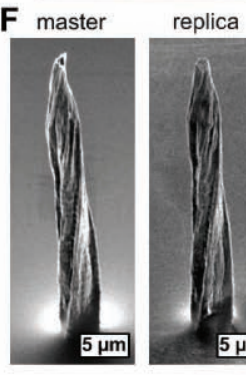

master

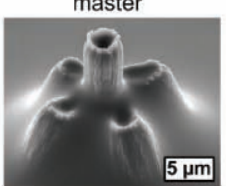

negative

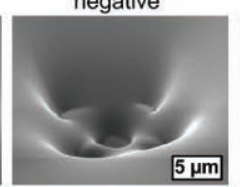

replica of $5^{\text {th }}$ neg.
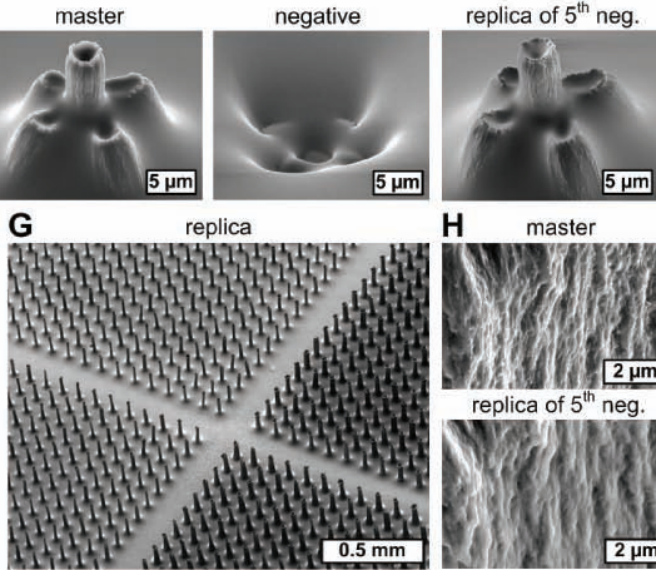

H master

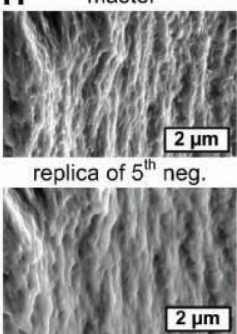

Figure 4. Mechanical properties and replica molding of capillary formed CNT structures. (A) Compression test schematic. (B) Exemplary capillary formed CNT and composite microcylinders. (C) Stress-strain curves. (D) Replication process. (E) Two-tiered "flower" structure at each stage of the fabrication sequence. (F) Master and SU-8 replica of helical pin. (G) Large array of thin-walled SU-8 microtubes replicated from CF-CNTs. (H) SEM images of sidewall surface texture of matching master and replica.

yet reported for CNT-polymer composite microstructures, and are notably higher than the value of $E=12 \mathrm{GPa}$ obtained by immersion of as-grown CNT forests in SU-8 in previous work. ${ }^{[12]}$ Therefore, our new two-step approach to densify the CNTs by capillary forming prior to polymer infiltration thus achieves a higher CNT packing density, avoids distortion of the as-grown CNT forests by polymer infiltration, and enables formation of unique 3D composite shapes over large areas.

Finally, CF-CNT-polymer composites can be used as master molds for mass-production of 3D polymer microstructures, as illustrated in Figure 4D. We created a variety of SU-8 replicas of 3D CNT structures including folded "flowers" (Figure 4E), helical pins (Figure 4F), and thin-walled tubes (Figure 4G). The mechanical integrity of the CF-CNT master gives repeated highfidelity replication of both microscale form and sub-micron surface topography, over multiple cycles (Figure $4 \mathrm{H}$ ). The ability to spatially vary the geometry and related properties of individual structures using capillary formed master molds represents an important new degree of design freedom for cost-effective fabrication of polymer microstructures. For example, arrays of polymer microstructures are widely used to study cell growth, differentiation, and mechanotransduction. ${ }^{[23]}$

By exploiting a local and deterministic transformation of vertically aligned nanostructures, capillary forming enables the design, fabrication, and integration of novel 3D microassemblies and complex surface architectures having spatially varying geometries, anisotropic textures, and multifunctional properties. This methodology can be extended from CNTs to other nanostructures made by natural or engineered methods, such as semiconducting or piezoelectric nanowires, or biological filaments. Further progress may enable fabrication of structures with critical dimensions equaling those of a small number of filaments such as CNTs. Capabilities to modify CNT surfaces ${ }^{[24]}$ suggest it will be possible to integrate advanced chemical and biological functionalities, while retaining complex and robust 3D shapes.

\section{Experimental Section}

CNT growth: CNT forest microstructures are fabricated on thermallyoxidized (100) silicon wafers on which $10 \mathrm{~nm}$ of $\mathrm{Al}_{2} \mathrm{O}_{3}$ and $1 \mathrm{~nm} F$ are sequentially deposited by e-beam evaporation. The catalyst is patterned by liftoff using photoresist SPR 220 and ultrasonic agitation in acetone. Next, CNT forests are grown in a horizontal tube furnace (22 $\mathrm{mm}$ ID, $300 \mathrm{~mm}$ heated length) at atmospheric pressure, with flows of $100 / 400 / 100 \mathrm{sccm} \mathrm{C}_{2} \mathrm{H}_{4} / \mathrm{H}_{2} / \mathrm{He}$, at $775^{\circ} \mathrm{C}$. [12] The vertical growth rate is $\approx 100 \mu \mathrm{m} /$ minute, and the growth time is typically $0.5-5$ minutes depending on the desired forest height. The CNTs are rapidly cooled in the growth atmosphere before purging the CVD chamber with $\mathrm{He}$ (at below $100^{\circ} \mathrm{C}$ ). The top "crust" of the forest is optionally removed by etching in $\mathrm{O}_{2}$ plasma using a Plasmatherm 790 with $10 \mathrm{sccm} \mathrm{O}_{2}$ at $75 \mathrm{mTorr}$ and $80 \mathrm{~W}$ for 20-40 s.

Capillary forming: After CNT growth, the substrate is attached to an aluminum mesh and is inverted over a beaker (Figure S1) containing boiling acetone, which is located in a fume hood. Approximately 1 second of vapor exposure was used for every $50 \mu \mathrm{m}$ of CNT length (forest height). Using a large ( $1 \mathrm{~L}$ ) beaker with a small volume of liquid $(\approx 20 \mathrm{~mL})$ creates a uniform upward vapor flux, and keeps the substrate at a lower temperature that drives condensation of the liquid on the substrate.

Fabrication and replication of composite master molds: CF-CNT structures are spin-coated with SU8-2002 (Microchem) at $3000 \mathrm{rpm}$ for $60 \mathrm{~s}$. Then the SU-8 is pre-baked on a hot plate for 2 minutes at $65{ }^{\circ} \mathrm{C}$, cured by UV exposure using a Dymax 2000 EC Flood Lamp $\left(75 \mathrm{~mW} / \mathrm{cm}^{2}, 20 \mathrm{~s}\right)$, and post-baked for $4 \mathrm{~min}$ at $95^{\circ} \mathrm{C}$. Then, a negative mold of the structure D-CNT/SU-8 master is made by casting PDMS over the master, vacuum degassing, and curing for $9 \mathrm{~min}$ on a hot plate at $120^{\circ} \mathrm{C}$. After curing, the PDMS negative is manually removed from the master. Replication is performed by casting SU-8 2002 onto the PDMS negative. The SU-8 cast is cured by baking on a hot plate at $120^{\circ} \mathrm{C}$ for $14 \mathrm{~h}$, followed by UV flood exposure $\left(75 \mathrm{~mW} / \mathrm{cm}^{2}, 20 \mathrm{~s}\right)$. The PDMS is manually removed from the replica. 


\section{Supporting Information}

Supporting Information is available from the Wiley Online Library or from the author.

\section{Acknowledgements}

M.D.V. and S.H.T. contributed equally to this work. This research was supported by the College of Engineering and Department of Mechanical Engineering at the University of Michigan, the Belgium Fund for Scientific Research - Flanders (FWO), and the Nanomanufacturing program of the National Science Foundation (CMMI-0927634). We thank John Mansfield for assistance with electron microscopy, Weidian Shen for assistance with nanoindentation, Zhaohui Zhong for assistance with electrical characterization, and Joerg Lahann for insightful discussions.

Received: April 12, 2010

Revised: June 14, 2010

Published online: September 2, 2010

[1] J. K. Gansel, M. Thiel, M. S. Rill, M. Decker, K. Bade, V. Saile, G. von Freymann, S. Linden, M. Wegener, Science 2009, 325, 1513.

[2] N. Blow, Nat. Methods 2009, 6, 619.

[3] A. Tuteja, W. Choi, J. M. Mabry, G. H. McKinley, R. E. Cohen, Proc. Natl. Acad. Sci. USA 2008, 105, 18200; J. Lahann, R. Langer, MRS Bull. 2005, 30, 185.

[4] M. A. Unger, H. P. Chou, T. Thorsen, A. Scherer, S. R. Quake, Science 2000, 288, 113.

[5] A. Bertsch, S. Jiguet, P. Renaud, J. Micromech. Microengin. 2004 14, 197; J. Stampfl, S. Baudis, C. Heller, R. Liska, A. Neumeister, R. Kling, A. Ostendorf, M. Spitzbart, J. Micromech. Microengin. 2008, 18, 125014.

[6] a) C. N. LaFratta, J. T. Fourkas, T. Baldacchini, R. A. Farrer, Angew. Chem. Int. Ed. 2007, 46, 6238; b) S. Kawata, H. B. Sun, T. Tanaka, K. Takada, Nature 2001, 412, 697.

[7] S. Reyntjens, R. Puers, J. Micromech. Microengin. 2001, 11, 287.

[8] A. del Campo, C. Greiner, J. Micromech. Microengin. 2007, 17, R81.

[9] G. M. Whitesides, B. Grzybowski, Science 2002, 295, 2418.
[10] a) D. A. W. Thompson, On Growth and Form 1917; b) J. Aizenberg, I. C. Weaver, M. S. Thanawala, V. C. Sundar, D. E. Morse, P. Fratz Science 2005, 309, 275.

[11] a) P. W. K. Rothemund, Nature 2006, 440, 297; b) K. J. M. Bishop C. E. Wilmer, S. Soh, B. A. Grzybowski, Small 2009, 5, 1600.

[12] A. J. Hart, A. H. Slocum, J. Phys. Chem. B 2006, 110, 8250.

[13] K. Hata, D. N. Futaba, K. Mizuno, T. Namai, M. Yumura, S. Iijima, Science 2004, 306, 1362.

[14] J. Bico, B. Roman, L. Moulin, A. Boudaoud, Nature 2004, 432, 690.

[15] C. Py, R. Bastien, J. Bico, B. Roman, A. Boudaoud, Europhys. Lett. 2007, 77

[16] B. Pokroy, S. H. Kang, L. Mahadevan, J. Aizenberg, Science 2009 323, 237.

[17] N. Chakrapani, B. Q. Wei, A. Carrillo, P. M. Ajayan, R. S. Kane, Proc Natl. Acad. Sci. USA 2004, 101, 4009.

[18] a) H. Liu, S. H. Li, J. Zhai, H. J. Li, Q. S. Zheng, L. Jiang, D. B. Zhu, Angew. Chem. Int. Ed. 2004, 43, 1146; b) M. A. Correa-Duarte, N. Wagner, J. Rojas-Chapana, C. Morsczeck, M. Thie, M. Giersig, Nano Lett. 2004, 4, 2233.

[19] a) D. N. Futaba, K. Hata, T. Yamada, T. Hiraoka, Y. Hayamizu, Y. Kakudate, O. Tanaike, H. Hatori, M. Yumura, S. lijima, Nat. Mater. 2006, 5, 987; b) D. N. Futaba, K. Miyake, K. Murata, Y. Hayamizu, T. Yamada, S. Sasaki, M. Yumura, K. Hata, Nano Lett. 2009, 9, 3302.

[20] Y. Hayamizu, T. Yamada, K. Mizuno, R. C. Davis, D. N. Futaba, M. Yumura, K. Hata, Nat. Nanotechnol. 2008, 3, 289.

[21] D. N. Futaba, K. Hata, T. Yamada, T. Hiraoka, Y. Hayamizu Y. Kakudate, O. Tanaike, H. Hatori, M. Yumura, S. lijima, Nat. Mater. 2006, 5, 987.

[22] a) E. J. Garcia, A. J. Hart, B. L. Wardle, A. H. Slocum, Adv. Mater. 2007, 19, 2151; b) J. N. Coleman, U. Khan, W. J. Blau, Y. K. Gun'ko, Carbon 2006, 44, 1624

[23] a) S. Raghavan, C. S. Chen, Adv. Mater. 2004, 16, 1303; b) F. Guilak D. M. Cohen, B. T. Estes, J. M. Gimble, W. Liedtke, C. S. Chen, Cell Stem Cell 2009, 5, 17.

[24] D. Tasis, N. Tagmatarchis, A. Bianco, M. Prato, Chem. Rev. 2006 106, 1105

[25] The densification factor is defined as the ratio of the initial crosssectional area of the CNT forest to the cross-sectional area at the midplane of the structure after capillary forming. 